\title{
Obesidade e Distúrbio Respiratório do Sono, Uma Associação de Fatores de Risco
}

S

ER DO SEXO MASCULINO E estar acima do peso são os principais fatores $\checkmark$ de risco para a síndrome da apnéia e hipopnéia obstrutiva do sono (SAHOS). Entretanto, obesidade e sobrepeso têm sido considerados agravantes e não determinantes da síndrome (1). Tendo em vista a epidemia de obesidade em vários países ocidentais, estima-se que a prevalência de SAHOS seja maior do que a anteriormente estimada. A SAHOS é conhecida por aumentar o risco cardiovascular e por ser fator de risco independente para hipertensão arterial (2). Recentemente, essa síndrome tem sido também implicada na patogênese da alteração do metabolismo da glicose (3). Aumento do esforço respiratório, hipóxia intermitente e fragmentação do sono podem levar a uma cascata de eventos incluindo alterações autonômicas, especialmente aumento do tônus simpático, alterações da função neuroendócrina e liberação de potentes agentes inflamatórios como o TNF-alfa e interleucina $(1,2)$. Além disso, alteração da leptina tem sido descrita como secundária à SAHOS, e alguns especulam que isso poderia, em parte, explicar a dificuldade que esses pacientes apresentam em perder peso, antes de serem adequadamente tratados com o CPAP nasal (pressão positiva contínua na VAS) (1). Por outro lado, a obesidade por si só pode determinar o aparecimento de hipertensão, inflamação, lesão endotelial e dislipidemia, justificando a maior mortalidade e morbidade observada nesse grupo de pacientes (4) e contribuindo para a evolução desfavorável dos pacientes com SAHOS. Esses dados reforçam o conceito de que o distúrbio respiratório do sono deve ser investigado em pacientes obesos. Os sintomas apresentados pelos obesos têm pouca capacidade de prever a SAHOS. O melhor preditor ainda é considerado a observação dos eventos pelos familiares (5). A polissonografia é o exame de escolha para o diagnóstico apropriado. O diagnóstico precoce pode determinar melhor prognóstico ao paciente (6). $\mathrm{O}$ artigo "Apresentação Clínica de Pacientes Obesos com Diagnóstico Polissonográfico de Apnéia Obstrutiva do Sono", de Gregório e cols. (7), avaliou todos os pacientes obesos (IMC $\geq 30 \mathrm{Kg} / \mathrm{m}^{2}$ ) que realizaram polissonografia em dois laboratórios de sono, que apresentaram IAH (índice de apnéia e hipopnéia) maior ou igual a 5 eventos por hora. Os sintomas avaliados foram sonolência excessiva diurna, despertares noturnos e sensação de engasgo ou sufocamento durante o sono. Os autores encontraram uma alta freqüência de SAHOS grave (IAH > 30) nessa população, não tendo sido observada diferença significativa em relação à gravidade da doença entre indivíduos com e sem sintomas. A freqüência de pacientes assintomáticos foi maior no grupo com obesidade II e III.

Os autores concluem que a polissonografia é fundamental na avaliação de pacientes obesos com apnéia do sono. Tais dados estão em acordo com a literatura prévia. Entretanto, a maior parte dos estudos, incluindo o de Gregório e cols., não avalia a presença de hipoventilação alveolar durante o sono, uma vez que medir o $\mathrm{CO}_{2}$ não faz parte da rotina da polissonografia do adulto. Resta, portanto, avaliar o quanto a

\section{editorial}

\section{Dalva Poyares \\ WALTER MORAES}

Médica Neurologista e

Professora Afiliada do

Departamento de Psicobiologia da Universidade Federal de São

Paulo/Escola Paulista de

Medicina - UNIFESP/EPM, São

Paulo, SP;

Médico Pesquisador da

Disciplina de Medicina e

Biologia do Sono, Departamento

de Psicobiologia da

Universidade Federal de São

Paulo/ Escola Paulista de

Medicina - UNIFESP/EPM, São

Paulo, SP. 
hipoventilação alveolar durante o sono, associada ou não a apnéia e hipopnéia obstrutiva do sono, influi na evolução desfavorável de pacientes obesos.

\section{REFERÊNCIAS}

1. Vgontzas AN, Papanicolaou DA, Bixler EO, Hopper K, Lotsikas $A$, Lin HM, et al. Sleep apnea and daytime sleepiness and fatigue: relation to visceral obesity, insulin resistance, and hypercytokinemia. J Clin Endocrinol Metab 2000; 85(3):1151-8.

2. Cintra FD, Poyares D, Guilleminault C, Carvalho AC, Tufik S, de Paola AA. Cardiovascular comorbidities and obstructive sleep apnea. Arq Bras Cardiol 2006;86(6):399-407.

3. Punjabi NM, Polotsky VY. Disorders of glucose metabolism in sleep apnea. J Appl Physiol 2005;99(5):1998-2007.

4. Huber C, Hunsaker DM, Hunsaker JC. The relationship between elevated body mass index and lethal ischemic heart disease: an eleven-year retrospective review of medical examiners' adult autopsies in Kentucky. J Ky Med Assoc 2005;103(3):93-101.
5. Dixon JB, Schachter LM, O'Brien PE. Predicting sleep apnea and excessive day sleepiness in the severely obese: indicators for polysomnography. Chest 2003;123(4):1134-41.

6. O'Keeffee T, Patterson EJ. Evidence supporting routine polysomnography before bariatric surgery. Obes Surg 2004:14:23-6.

7. Gregório PB, Athanazio RA, Bitencourt AGV, Neves FBCS, Daltro C, Alves E, et al. Apresentação clínica de pacientes obesos com diagnóstico polissonográfico de apnéia obstrutiva do sono. Arq Bras Endocrinol Metab 2007; 51/7:1065-8.

\section{Endereço para correspondência:}

Dalva Poyares

Rua Marselhesa 524

04020-060 São Paulo, SP

Fax: (1 1) 5572-5092

E-mail: poyares@psicobio.epm.br 